(Aus der Universitäts-Augenklinik zu Würzburg.)

\title{
Experimentelle und kritische Untersuchungen zur Frage nach dem Einfluss des Nervus sympathicus auf den Accommodationsvorgang.
}

\author{
Von \\ Dr. Paul Römer, und Othmar Dufour, \\ Privatdocent, Volontärassistent der Klinik.
}

Die Anschauung, dass der Nervus sympathicus einen Einfluss auf den Accommodationsvorgang ausübe, taucht von Zeit zu Zeit immer einmal wieder in der ophthalmologischen Literatur auf.

So machten Morat und Doyon im Jahre 1891(1) die Angabe, dass nach Durchschneidung des Sympathicus von ihnen eine Verkleinerung, bei Reizung desselben eine Vergrösserung des vorderen Linsenbildchens gesehen worden sei. Sie zogen aus ihren Untersuchungen den Schluss, dass die Reizung des Sympathicus eine Abflachung der Linse und damit eine Einstellung des Auges für entfernte Objecte zur Folge habe. Sie glauben, dass es sich dabei um eine hemmende Wirkung des Sympathicus auf die Ciliarmuskelcontractionen handele und sprechen direct den Sympathicus als Hemmungsnerv für die Accommodation an. Nach ihnen giebt es nicht einen, sondern zwei Nerven für die Accommodation: „L'Oculomoteur, qui adapte l'oeil pour la vision des objets rapprochés et le grand sympathique, qui l'adapte pour la vision des objets eloignés (Arch. de Physiologie 1891. p. 509).

Ein derartiger Einfluss des Sympathicus auf die Formgestaltung der Linse war dagegen von Langley und Andersen(2) in Abrede gestellt worden, nachdem bereits Hensen und Völkers im Jahre $1868 \mathrm{zu}$ demselben Resultat gekommen waren.

Zur Entscheidung dieser Frage hatte dann Hess mit Heine zusammen Versuche angestellt, deren Resultate der Morat-Doyonschen Hypothese direct widersprachen. 
Hess reizte zunächst bei einem Hunde mittels local am Auge angewandter schwacher Ströme den Ciliarmuskel. Die Wirkung der Contraction desselben liess sich an der Bewegung einer im Aequator des Bulbus eingestochenen Nadel feststellen. Wurde nun während dieser Reizung des Ciliarmuskels gleichzeitig der freigelegte Sympathicus mit Strömen der verschiedensten Stärke gereizt, so erweiterte sich zwar die Pupille sehr prompt, die Nadel blieb aber unbewegt in der dem contrahirten Ciliarmuskel entsprechenden Stellung.

Es ergab sich also aus diesem Versuch, dass der Sympathicus wohl die Pupille erweitern, nicht aber den Ciliarmuskel beeinflussen konnte.

In einem zweiten Versuche an einem Hunde beobachteten Hess und Heine, während sich bei Reizung des Sympathicus die Pupille stark erweiterte, eine Refractionsverminderung um 1 bis 1,5 D. Eine im Aequator bulbi eingestochene Nadel blieb auch in diesem Versuch von der Sympathicusreizung völlig unbeeinflusst.

Hess machte schon damals darauf aufmerksam, dass die bei Erweiterung der Pupille beobachtete Refractionsverminderung um 1 bis $1,5 \mathrm{D}$ nicht nothwendig auf negative Accommodation zu beziehen sei, sondern durch den Einfluss der peripheren Hornhaut- und Linsenpartien auf die Refraction bedingt sein könnte.

Morat und Doyon verengten zunächst die Pupille künstlich durch Eserin oder Nicotin: Pour rendre son effet plus visible, il est bon de produire au prealable le spasme de l'accommodation, soit par l'excitation du nerf antagoniste, soit par l'instillation dans l'oeil de substances myotiques auxquelles nous avons reconnu un effet parallele et de même ordre sur le systeme accommodateur, l'eserine ou préferablement la nicotine, à la dose d'une goutte de la solution $1 / 100^{*}$ (Comptes rendus des seances de l'Academie des sciences 1891. p. 1328.)

Bei so enger Pupille wird es aber, wie wir uns überzeugt haben, nur durch besondere, verhältnissmässig schwer zu erfiillende Vorsichtsmassregeln überhaupt erreicht, dass das ganze vordere Linsenbildchen in den mittleren Pupillentheilen so zur Anschauung kommt, dass sicher nicht ein kleiner Theil des bekanntlich ja immer sehr verwaschenen Bildes in seinen Randpartien von der Iris verdeckt. wird. Wird das Linsenbildchen erst bei Erweiterung der Pupille in toto sichtbar, so wird eine Vergrösserung desselben auch dadurch vorgetäuscht werden, dass nun die peripheren weniger stark gewölbten Linsenpartien an seinem Zustandekommen mitwirken können. Bei Morat und Doyon finden sich keinerlei Angaben darüber, ob und 
Experim. u. krit. Untersuch, zur Frage nach d. Einfluss d. Nervus sympath. etc. 493

in welcher Weise auf diese Fehlerquelle Rücksicht genommen wurde. Die von Morat und Doyon in den Vordergrund gestellte Beobachtung, dass bei Reizung des Sympathicus sich das vordere Linsenbildchen vergrösserte, kann daher auf Grund dieser rein optischen Verhältnisse nicht als Beweis für eine durch Sympathicusreizung eintretende Abflachung der vorderen Linsenfläche angesehen werden.

Die Hauptversuche von Morat and Doyon konnten also nach Hess und Heine ohne die Annahme einer accommodativen Abflachung der Linse erklärt und der Einfluss des Sympathicus auf die Accommodation nach ihren eigenen Versuchen in Abrede gestellt werden.

So stand die Frage, als Dor (4) auf dem Pariser Congress im Anschluss an das Referat von Hess über den gegenwärtigen Stand der Acommodationslehre auf die Versuche von Morat und Doyon zurückgriff und angab, auch durch die Versuche von Hess und Heine sei die Frage nach dem Einfluss des Sympathicus auf die Accommodation noch nicht erledigt. Vor Allem sprächen zwei Momente für die Auffassung von Morat und Doyon: Einmal die Beobachtung, dass nach Reizung des Sympathicus von Hess und Heine selbst skiaskopisch eine Refractionsverminderung festgestellt sei. Und zweitens beruft sich Dor auf folgenden seiner Ansicht nach wichtigen Versuch von Morat und Doyon:

"Chien, taille au-dessus de la moyenne, morphine 5 centigrammes en injection dans le tissu cellulaire - chloroforme - isolement d'un nerf ciliaire, on l'excite, la pupille se contracte, l'image cristallinienne antérieure qui était environ de la grandeur de l'image cornéenne devient beaucoup plus petite. On excite ensuite le sympathique au cou en anse, du côté correspondant la pupille se dilate, l'image grandit; on excite un autre nerf ciliaire, l'image devient petite; en même temps que ce nerf ciliaire est excité on excite le sympathique correspondant au cou; on voit alors la pupille rester contractée et l'image grandir beaucoup." (Thèse de Doyon, Masson 1891. p. 45.)

Angesichts dieser Finwände und der grossen Bedeutung des Problems für die Accommodationslehre hat uns Herr Prof. Hess beauftragt, die Frage nach dem Einfluss des Sympathicus auf den Accommodationsvorgang von Neuem zu untersuchen.

Was zunächst den ersten von Dor hervorgehobenen Punkt, die skiaskopisch festgestellte Refractionsabnahme um 1 bis $1,5 \mathrm{D}$ bei Sympathicusreizung angeht, so muss noch einmal betont werden, dass es nicht zulässig ist, aus derselben auf eine durch Reizung jenes Nerven herbeigeführte Abflachung der vorderen Linsenfläche zu schliessen. Morat und Doyon sagen bezüglich der Grössenzunahme des vorderen Linsenbildchens: "Ce qu'il importe de savoir, c'est que 
la valeur de ce grandissement est telle, qu'il ne peut y avoir aucun doute sur son existence réelle et que le sens de sa variation est constant. Il nous a paru souvent que l'image s'agrandissait entiron d'un tiers, d'une moitié de son diamètre: une fois sur un chat, l'image nous a paru presque doubler" (Arch. de Physiol. 1891. p. 511).

Auf die Schwierigkeit, mittels einfacher Beobachtung derartige Grössendifferenzen im wechselnden Verhalten des Linsenbildchens zu schätzen, hat Hess bereits hingewiesen. Aber selbst wenn jene Grössenunterschiede von Morat und Doyon richtig bestimmt sind, so steht nach Berechnungen, die uns Herr Prof. Hess an die Hand gegeben hat, eine derartige Grössenzunahme des vorderen Linsenbildchens, wie sie Morat und Doyon angeben, mit dem erwähnten skiaskopischen Befund der Refractionsverminderung im Widerspruch. Denn da die Grösse des Bildchens dem Radius der Krümmungsfläche direct proportional ist, so müsste bei einer Zunahme der Bildgrösse auf das Doppelte der ursprünglichen Grösse der Linsenradius etwa doppelt so gross geworden sein, was einer jedenfalls viel grösseren Refractionsverminderung entsprechen müsste, als sie beobachtet ist. Es sei daran erinnert, das nach Helmholtz beim Menschen eine Aenderung des Radius von 6 auf $10 \mathrm{~mm}$ - wobei also das Linsenbildchen nur etwa um zwei Drittel grösser wird - einer Refractionsverminderung um $7 \mathrm{D}$ entspricht. Thatsächlich haben aber Hess and Heine bei dem ron ihnen untersuchten Hunde nur eine Refractionsabnahme von 1 bis $1,5 \mathrm{D}$ constatiren können.

Schon nach dieser Berechnung erscheint die Schlussfolgerung von Morat und Doyon, dass bei Sympathicusreizung die Vergrösserung des vorderen Linsenbildchens auf der Abflachung der vorderen Linsenfläche beruhe, ziemlich unsicher. Ueber die genaueren entsprechenden Werthe der Hornhaut- und Linsenkrümmung bei Thieren wissen wir bis jetzt nichts. Wenn sie jenen beim Menschen ähnlich sind, so könnte unter gewissen Umständen bei Erweiterung der $\mathrm{Pu}$ pille eine Grössenzunahme des Linsenbildchens um das Doppelte eintreten, ohne dass die Wölbung der Linsenvorderfläche sich im Geringsten geändert zu haben braucht. Eine Grössenzunahme des vorderen Linsenbildehens bei Reizung des Sympathicus ist also noch kein Beweis für eine durch denselben herbeigeführte Abflachung der vorderen Linsenfläche.

Ebenso wenig wie die Beobachtung des vorderen Linsenbildchens kann weiterhin der von Dor angeführte und oben bereits citirte Versuch von Morat und Doyon den Einfluss des Sympathicus auf den 
Accommodationsvorgang beweisen. In jenem Versuche erfolgte zuerst nach der Reizung eines Ciliarnerven Contraction der Pupille und Verkleinerung des Linsenbildchens. Darauf wurde der Sympathicus gereizt, die Pupille erweiterte sich, das Linsenbildchen vergrösserte sich. Darauf wurde ein anderer Ciliarnerv gereizt, und wieder wurde bei Pupillenverengerung das Bild kleiner. Als nun aber gleichzeitig der Sympathicus gereizt wurde, sahen die Autoren alsdann die Pupille im Contractionszustand verharren und das Linsenbildchen grösser werden. Wenn in einem derartigen Versuch nach Reizung des Sympathicus die Pupille eng bleibt, so ist die Controle, ob überhaupt eine Reizuibertragung durch den Nerven erfolgt ist, verloren gegangen. Denn der einzige thatsächliche Anhaltspunkt dafür, dass die Reizung des Sympathicus bei dem fraglichen Versuch einen Effect gehabt hat, ist bis jetzt ja die Erweiterung der Pupille. Auch dieser Versuch, auf den Dor so grosses Gewicht legt, muss daher aus der Verwerthung für die Morat-Doy on'sche Hypothese ausgeschaltet werden. Wollte man denselben verwerthen, so müsste man ja schliessen, dass der Sympathicus nur auf die Accommodation, nicht aber auf die Pupille wirke, was nicht wohl angängig ist. (Dass Morat und Doyon selbst kein sehr grosses Gewicht auf ihn gelegt haben, scheint daraus hervorzugehen, dass dieser Versuch nur in der Thèse von Doyon, nicht aber in ihrer Arbeit im Arch. de Physiol. angeführt ist.)

Dagegen haben Morat und Doyon über ein Versuchsresultat an zwei Hunden berichtet, welches, wenn es sich bestätigt, für die Frage nach der Rolle des Sympathicus bei der Accommodation grössere Bedeutung haben würde, als die unsichere Beobachtung der Grössenzunahme des Linsenbildchens bei den besprochenen Versuchen:

,Dans deux expériences sur le chien, nous avons introduit à travers le bord de la cornée une aiguille dont la pointe s'arrêtait au centre de la face antérieure du cristallin. Cette aiguille se prolongeait au dehors sous la forme d'un levier amplifié à longue branche extérieure et qui était équilibré de manière à appuyer légèrement sur le cristallin afin de ne jamais quitter sa surface. L'excitation du sympathique modifiait la position de ce levier de telle façon qu'elle indiquait toujours un abaissement de sa courte portion vers le fond de l'oeil, autrement dit un aplatissement du cristallin" (Arch. de Physiol. 1891. p. 512).

Zur Nachprüfung diẻser Angaben haben wir zunächst eine Reihe von Versuchen an Kaninchen ausgeführt, von denen einer kurz beschrieben sei: 
In Aethernarkose wurde der linke Halssympathicus freigelegt, die Reizung desselben mittels Inductionsstromes ergab prompte Erweiterung der Pupille auf der entsprechenden Seite. Nachdem der Sympathicus vor Vertrocknung geschützt war, wurde das linke Auge nach Entfernung der Lider und Abtrennung der äusseren Augenmuskeln so weit frei präparirt, dass selbst starke, local am Auge angewandte Ströme keinerlei Bewegung desselben herbeizuführen vermochten. Nun wurde eine Nadel durch die Sklera in einer Entfernung von $4 \mathrm{~mm}$ in den Ciliarmuskel eingestossen. Die locale Reizung des Ciliarmuskels ergab bei diesem Thier die bekannte Bewegung der Nadel.

Nachdem dies festgestellt war, wurde eine zweite Nadel ron oben her so durch die Hornhaut gestochen, dass sie im Pupillargebiet der vorderen Linsenkapsel auflag. Bei Reizung des Sympathicus erfolgte jedes Mal prompte Erweiterung der Pupille, dagegen machte die auf der vorderen Linsenkapsel liegende Nadel keine Bewegung. Nachdem daher sowohl Ciliarmuskel als auch Sympathicus auf Reize prompt reagirten, gingen wir dazu über, die Beziehungen zwischen den Ciliarmuskelcontractionen und der Sympathicusreizung zu studiren.

Es wurde zuerst mit verschieden starken Strömen der Ciliarmuskel gereizt; die im Aequator bulbi steckende Nadel machte ihren Ausschlag und ging in die der Contraction des Muskels entsprechende Stellung.

Nun wurde mit Hilfe eines zweiten Inductionsapparates der Sympathicus, und zwar ebenfalls mit Strömen verschiedener Stärke gereizt: die Nadel auf der vorderen Linsenkapsel blieb völlig unbeweglich, während die Pupille sich prompt erweiterte.

Ebenso machte die im Ciliarmuskel steckende Nadel keine Bewegung. An dieser letzteren trat erst dann eine Bewegung, der Uebergang in die Ruhestellung, ein, wenn die Reizung des Ciliarmuskels unterbrochen wurde und nun die Nadel in ihre primäre Stellung zurückkehrte.

Dieser erste am Kaninchen angestellte Versuch zeigte also, dass von einer hemmenden Wirkung des Sympathicus auf den Ciliarmuskel und auf die Linse keine Rede war.

Im Einzelnen ergab sich bei unseren zunächst zur Orientirung über die Technils an vier Kaninchen angestellten Versuchen noch Folgendes, was bei der experimentellen Untersuchung dieser Frage der Beachtung werth ist.

Zunächst muss bei der Freilegung des Bulbus' sorgfältig darauf Rücksicht genommen werden, dass alle äusseren Augenmuskeln durch- 
trennt werden. Denn die geringsten Faserverbindungen können leicht durch Bewegung des Auges zu Täuschungen uber etwaige Nadelbewegungen Veranlassung geben.

Ferner ist der Effect der Ciliarmuskelreizung bei Kaninchen ein sehr verschiedener, da dieser Muskel bei ihnen sehr mangelhaft ausgebildet ist. So ist es uns unter vier Thieren nur einmal gelungen, eine einwandsfreie Bewegung der Ciliarmuskelnadel bei localer Reizung zu erzielen.

Ebenso haben unsere Versuche über die Einführung der Nadeln in die vordere Kammer auf die Vorderfläche der Linse ergeben, dass hier mancherlei zu beachten ist. Vor Allem ist darauf zu sehen, dass die Spitze der Nadel so im Centrum des Pupillengebietes auf der Linsenkapsel liegt, dass bei Verengerung der Pupille die Nadel nicht mit der Iris in Berührung kommt. Letzteres ist aber fast unvermeidlich, wenn man die Nadel durch den Hornhautrand einführt, wie dies Morat und Doyon gethan haben. Wir haben, um diesen Fehler zu vermeiden, die Nadel stets ungefähr in der Mitte zwischen Hornhautmitte und Hornhautrand durchgestossen. Das hat den Vortheil, dass die Hornhaut hier dünner, der Stichcanal kürzer und in Folge dessen die Nadel leichter beweglich ist. Die Nadel wurde ferner immer ron oben her durch die Cornea eingestochen, damit sie sich durch ihre eigene Schwere an die Linse anlegt. Wir haben diese Anlagerung an die Linsenkapsel zuweilen noch dadurch verstärkt, dass. wir bei verschiedenen Versuchen die Nadel an ihrem langen, aus dem Auge herausragenden Ende mit einem feinsten Reiterchen beschwerten.

Wird der Sympathicus gereizt, wenn die Iris mit der Nadel in Berührung ist, so kann natürlich bei Frweiterung der Pupille sehr leicht eine Bewegung eintreten.

Aber die Nadel braucht nicht einmal direct die Iris zu berühren. Sobald der Versuch längere Zeit ausgedehnt wird, kommt es in dem Kammerwasser des Kaninchens leicht zu Gerinnungen. Es bedarf dann nur, wie wir uns überzengt haben, einer zartesten Faserverbindung zwischen Nadel und Iris, um bei Bewegung der Pupille in Folge Sympathicusreizung eine Bewegung der Nadel auszulösen.

Gegen diese bisher besprochenen Versuche kann der Einwand erhoben werden, dass der Accommodationsmechanismus beim Kaninchen so mangelhaft ausgebildet sei, dass eine hemmende Wirkung des Sympathicus nicht mit Sicherheit festgestellt werden kann. Aber alle diese Fehlerquellen mussten erst studirt werden, bevor wir zum Versuch am Hunde ubergingen. 
Beim Hunde ist dieser Mechanismus genügend entwickelt, dass man ein Versagen der Ciliarmuskelcontraction nicht zu fürchten braucht.

In Aethernarkose wird bei einem kräftigen Hunde der linke Sympathicus am Halse freigelegt. Bis der Bulbus frei präparirt ist, wird der Nerv durch Klemmverschluss der Hautwunde vor Vertrocknen geschützt. Ebenso wird das Auge während des ganzen Versuches häufig mit warmer physiologischer Kochsalzlösung berieselt.

Am linken Bulbus selbst werden die Lider abgetragen, die äusseren Augenmuskeln von der Sklera abgetrennt. Starke Inductionsströme am Auge sind nicht mehr im Stande, eine Retractionsbewegung des Bulbus herbeizuführen, das Auge ist also hinreichend isolirt.

Nun wird oben im Aequator bulbi eine feine Insectennadel so eingestochen, dass man beim Blick durch die Pupille ihre Spitze eben erkennen kann.

Bei Reizung des Ciliarmuskels mittels ạm Auge applicirter Ströme von verschiedener Stärke macht die Nadel regelmässig grosse Auśschläge und die Pupille verengert sich. Bei dieser Reizung sind mit Hilfe eines Statives die Elektroden so festgestellt, dass ihre Spitzen den Bulbus leicht berühren. Diese in allen unseren Versuchen angewandte Vorsichtsmassregel ist absolut erforderlich, um jede Bewegung von Seiten des Experimentators auszuschliessen.

Nachdem so festgestellt war, dass der Ciliarmuskel prompt functionirte, wurde eine Nadel auch durch die Cornea so eingeführt, dass sie die vordere Linsenkapsel berührte. Auch in diesem Versuch wurde verschiedene Male die Nadel mit einem feinen Reiterchen beschwert, und theils mit theils ohne diese Belastung experimentirt. Bei Reizung des Sympathicus erweiterte sich die Pupille ausgiebig, die Nadel auf der vorderen Linsenfläche sowohl wie die im Ciliarmuskel steckende blieben unbeweglich.

Nachdem dies festgestellt war, konnte der Hauptrersuch ausgeführt werden:

Der Ciliarmuskel wird gereizt: die Pupille wird eng, die im Aequator bulbi steckende Nadel macht ihren Ausschlag und geht in die der Contraction entsprechende Stellung. Während diese Reizung des Ciliarmuskels fortdauert, wird jetzt der Sympathicus gereizt: die Pupille erweitert sich, die Nadel auf der vorderen Linsenkapel bleibt vollständig unbeweglich, ebenso tritt an der im Ciliarmuskel steckenden Nadel nicht die geringste Schwankung ein.

Jetzt wird der Strom im Sympathicus unterbrochen: die Pupille wird eng, die Nadeln bleiben unbeweglich. Dagegen kehrt bei Unter- 
brechung des Ciliarmuskelstromes die im Aequator steckende Nadel sofort in ihre primäre Stellung zurïck.

Der geschilderte Versuch zeigt einwandsfrei, dass der Sympathicus den Accommodationsvorgang nicht beeinflusst.

Es braucht kaum betont zu werden, dass wir auch bei diesem Versuche Ströme von verschiedener Stärke benutzt haben und zwischen den einzelnen Versuchen angemessene Pausen haben eintreten lassen. Das Resultat war bei häufiger Wiederholung stets das gleiche.

Aus unseren Untersuchungen geht hervor, dass die Angaben von Morat und Doyon nicht einwandsfrei sind und dass dem Sympathicus ein Einfluss auf den Accommodationsvorgang nicht zukommt.

Nach Abschluss dieser Untersuchungen erschien die Arbeit von Terrien und Camus (Arch. d'Ophtalm. 1902. Juin. p. 386). Diese Autoren beobachteten skiaskopisch bei Reizung des Sympathicus keine Abnahme, sondern eine Zunahme der Refraction (um 1 bis 2,5 D). Nach Angabe der Autoren sind diese Untersuchungen noch nicht abgeschlossen. Wir behalten uns vor, auf dieselben noch zurückzukommen.

Herm Prof. Hess sprechen wir für die Anregung zu diesen Untersuchungen und für die vielfache Unterstützung unseren ergebensten Dank aus.

\section{Literaturverzeichniss.}

1) Morat und Doyon, Le grand sympathique nerf accommodateur. Arch. de Physiol. 1891.

2) Langley and Andersen, On the mechanisme of the movement of the iris. Journ. of Physiol. XIII. 1892.

3) Hess und Heine, Arbeiten aus dem Gebiet der Accommodationslehre. v. Graefe's Arch. f. Ophthalm. Bd. XLVI.

4) Dor, Internat. med. Congress zu Paris 1900.

5) Hess, Internat. med. Congress zu Paris 1900. 